\title{
Optimization of a Broadband Gain Element for a Widely Tunable High-Power Semiconductor Disk Laser
}

\author{
Carl Borgentun, Jörgen Bengtsson, Anders Larsson, Senior Member, IEEE, Frank Demaria, Alexander Hein, and \\ Peter Unger, Member, IEEE
}

\begin{abstract}
The layer structure of the gain element in an optically pumped semiconductor disk laser was parametrically optimized with respect to a target function specifying a desired unsaturated reflectance over a desired wavelength range at a constant pump intensity. Spectral threshold pump intensity measurements confirmed the efficacy of the design, showing a much wider low-threshold regime than a conventional nonbroadband gain element, in good agreement with simulations. This evaluation avoids the possible influence of additional factors under high-power operation. Nonetheless, having a high and nearly constant broadband unsaturated reflectance at low pump intensity is a key to obtain good high-power performance, as evidenced by the obtained continuous tuning from 967 to $1010 \mathrm{~nm}$ with a maximum output power of $2.6 \mathrm{~W}$.
\end{abstract}

Index Terms-Birefringent filter (BRF), continuous tuning, high-power laser, InGaAs, optically pumped semiconductor disk laser (OP-SDL), vertical-external-cavity surface-emitting laser (VECSEL).

\section{INTRODUCTION}

$\mathbf{T}$ HE optically pumped semiconductor disk laser (OP-SDL), also referred to as the vertical-external-cavity surface-emitting laser (VECSEL), has the ability to provide high output power directly into a circular beam of high optical quality [1]-[5]. To obtain broadband tunability, one can use two or more separately pumped gain elements in the external cavity [6], each with a slightly different spectral reflectivity, but not without complicating the device. To obtain broadband tunability with a single gain element, it must be able to provide larger than unity reflectance over a wide wavelength range. This can be done by incorporating quantum wells (QWs) with different compositions or widths, thus having different gain

Manuscript received November 10, 2009; revised March 29, 2010; accepted April 02, 2010. Date of publication April 19, 2010; date of current version June 11,2010 . This work was supported in part by the Swedish Research Council under Grant 2006-2888.

C. Borgentun, J. Bengtsson, and A. Larsson are with the Photonics Laboratory, Department of Microtechnology and Nanoscience, Chalmers University of Technology, SE-412 96 Gothenburg, Sweden, (e-mail: carl.borgentun@chalmers.se;_jorgen.bengtsson@chalmers.se; anders. larsson@chalmers.se).

F. Demaria, A. Hein, and P. Unger are with the Institute of Optoelectronics, Ulm University, 89069 Ulm, Germany (e-mail: frank.demaria@uni-ulm.de; alexander.hein@uni-ulm.de; peter.unger@uni-ulm.de).

Color versions of one or more of the figures in this letter are available online at http://ieeexplore.ieee.org.

Digital Object Identifier 10.1109/LPT.2010.2048309

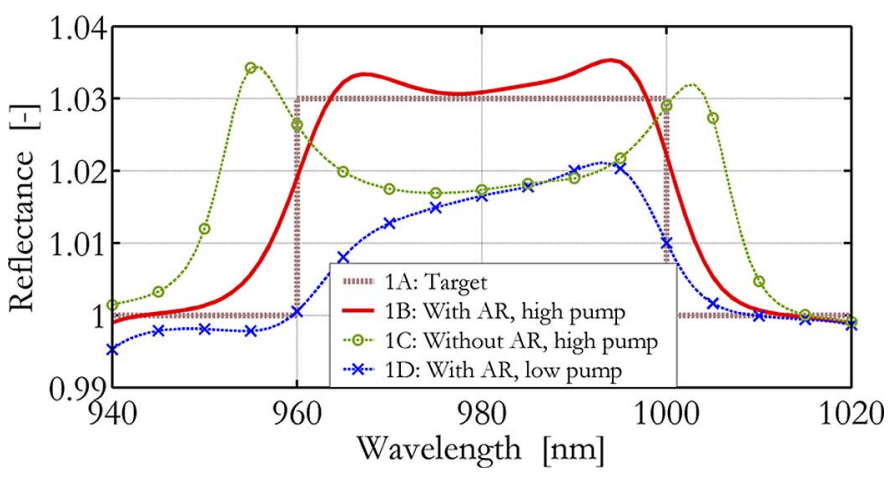

Fig. 1. Target unsaturated reflectance spectrum of the gain element for a cavity round-trip loss of $\sim 3 \%$ (1A) and simulated spectra for designed gain elements with (1B) and without (1C) an AR-structure. For the gain element using an AR-structure its reflectance is also shown at a lower pump intensity closely matching the threshold conditions in the experiments with $\sim 1.2 \%$ round-trip loss (1D).

spectra, into the element [7], or by using quantum-dot material with a broad gain spectrum [8]. It is also possible to use identical QWs, as in this work. Broadband performance is then obtained by deliberately making the subcavity formed between the air interface and the distributed Bragg reflector (DBR) antiresonant at the center wavelength. Thus as the wavelength is tuned, the smaller overlap between the antinodes of the standing wave field and the QWs is partly compensated by the increased field intensity in the entire subcavity as it becomes more resonant.

We use a strategy similar to [9], in which also an antireflection (AR) structure between the active region and the air interface is used, but we use a much longer active region with 12 QWs to absorb most of the pump light. For broadband performance, this complicates the optimization since the longer active region inevitably renders the performance more wavelength-dependent. Therefore, we allow for the AR structure to be parametrically optimized, which allows us to choose a target performance- the spectral reflectance at a certain low pump intensity without stimulated emission (i.e., the condition at threshold) — with which the actual simulated performance of the device is compared during optimization.

To experimentally verify the functionality of the designed gain element, ideally one would like to directly measure the same property as was optimized in the design-i.e., the unsaturated reflectance under constant pump intensity. Unfortunately, although conceptually simple, this would be a rather delicate 


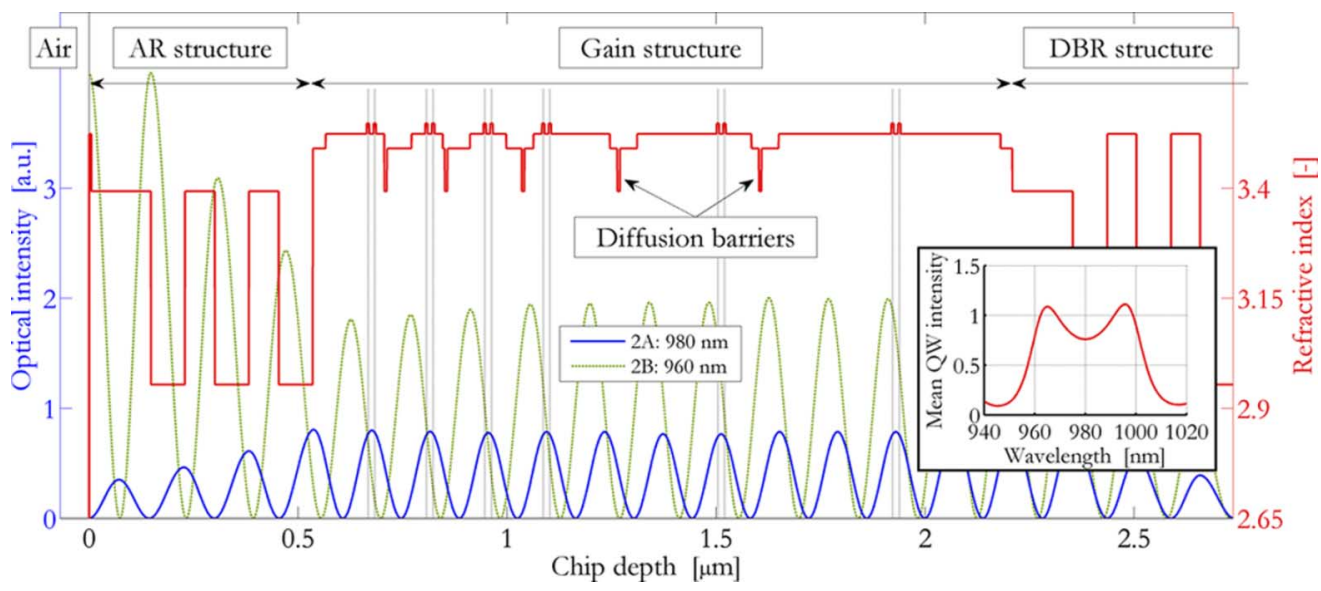

Fig. 2. Calculated intensity of the optical field inside the subcavity for the antiresonant (2A) and resonant (2B) wavelengths at 980 and $960 \mathrm{~nm}$, respectively. Also shown is the refractive index profile (solid red). The QWs are marked with vertical dotted black lines. The inset shows the average optical intensity in the QWs as a function of wavelength.

experiment involving a tunable laser probing the gain element surface, the latter being simultaneously pumped with an accurately controlled intensity. The commonly measured entity-the output power versus tuning wavelength - is a too indirect measure, since it also involves additional effects occurring at high pump power, mainly related to thermal issues. A simple experiment that still is very close to the direct measurement is to measure the spectral threshold pump intensity. This entity is also easily simulated using no other parameters than those used in the design, thus a direct comparison between experiment and the simulated performance of the optimized gain element is possible.

\section{Gain Element Design and Realization}

The target spectral reflectance for the gain element is shown in Fig. 1. Over the tuning range it should be constant and high enough to compensate for the assumed losses in the external cavity. In the gain element design, a transfer matrix model was used to calculate the field in the layer structure upon illumination from air with a unity intensity field, thus yielding the intensity of the reflected field and the effective reflectance. The gain in the QWs was calculated using a standard parabolic band model and one-dimensional quantum confinement with phenomenologically adjustable parameters to account for, e.g., strain, hole band splitting, and Coulomb interaction. The subcavity parameters that were optimized were the distribution of the QWs in the subcavity, the subcavity length, and the thicknesses, compositions, and number of layers in the AR structure.

To reduce the problem, the optimization was not fully global. The number of QWs was fixed and they were positioned in pairs at antinodes of the standing wave field at the center design wavelength of $980 \mathrm{~nm}$. To equally populate the QWs, high band-gap diffusion barriers were carefully positioned to account for the exponentially decaying pump intensity [10]. All these measures are illustrated in Fig. 2. Also, the subcavity length was chosen such that the field intensity in the subcavity was minimized at the center wavelength, i.e., the condition for an antiresonant subcavity, while simultaneously providing resonance, i.e., maximum field intensity, at 960 - and 1000-nm wavelengths ( $\pm 20 \mathrm{~nm}$ with respect to the center wavelength). The figure shows the subcavity fields for the 980- and 960-nm wavelengths; the inset shows the average intensity in the 12 QWs and illustrates how the resonances at 960 and $1000 \mathrm{~nm}$ compensate for the QW-antinode misalignment at these two wavelengths.

Under these design conditions for QW positioning and subcavity length, the thicknesses, chemical compositions, and number of layers in the AR structure were optimized to further balance the subcavity resonance effects to obtain a maximally flat and sufficiently high reflectance spectrum. Fig. 1 shows the simulated reflectance spectra after the optimization, with and without utilizing an AR structure.

For the physical realization we chose to use 12 identical $\operatorname{In}(0.20) \mathrm{Ga}(0.80) \mathrm{As}$ QWs of $6 \mathrm{~nm}$ width with a calculated gain peak at $980 \mathrm{~nm}$. The structure also included tensilely strained Ga-As-P layers containing 7\% phosphorus, which compensate for the compressive strain in the QWs. The AR structure consisted of 2.5 pairs of $\sim \lambda / 4$ thick $\mathrm{Al}-\mathrm{As} / \mathrm{Al}-\mathrm{Ga}-\mathrm{As}$ layers and one $\sim \lambda / 2$ thick $\mathrm{Al}-\mathrm{Ga}-\mathrm{As}$ layer.

\section{EXPERIMENTAL EVALUATION}

To ensure efficient heat removal, the structure was grown in reverse order and the metalized DBR was soldered directly to a copper submount. The substrate was then mechanically and chemically removed [11] leaving only the layers necessary for the gain element. The processed chips were mounted on a copper heat sink held at $-8{ }^{\circ} \mathrm{C}$ and used in a linear cavity (see the inset of Fig. 3). The outcoupling mirror had a reflectivity of $99 \%$ and a radius of curvature of $100 \mathrm{~mm}$. The main reason for choosing a higher mirror reflectivity than used in the design was to enable lasing in a larger wavelength range and thus obtain more data for the comparison between experiment and simulation (the simulation takes the higher reflectivity into account). Tunability was provided by a birefringent filter (BRF), a 1.0-mm-thick crystalline quartz plate, with a free spectral range of $\sim 80 \mathrm{~nm}$. The BRF was inserted in the cavity at the Brewster angle and was mounted such that it could be rotated around an axis parallel to the surface normal. 


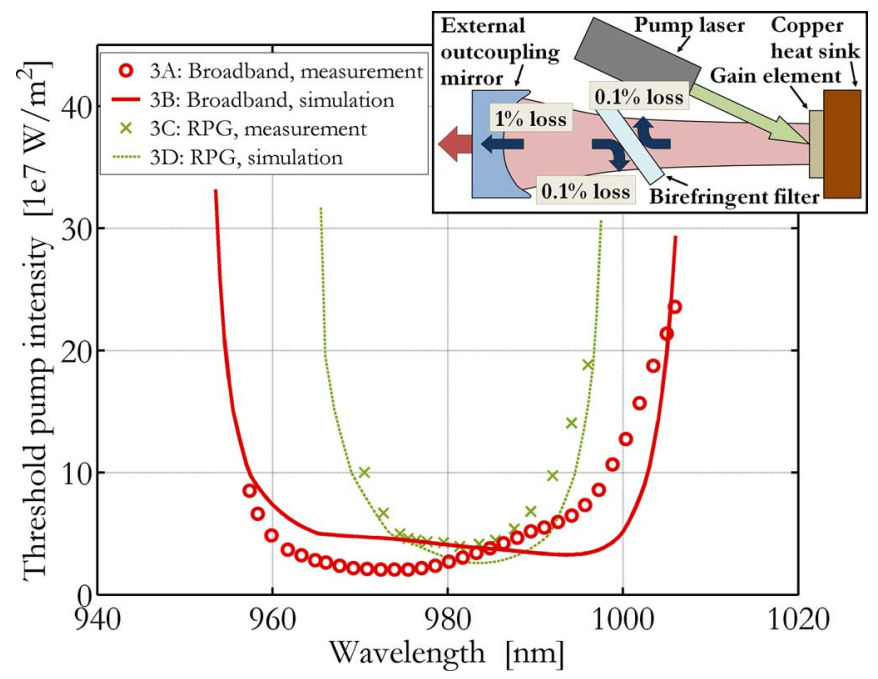

Fig. 3. Threshold pump intensity as a function of wavelength for the designed gain element (3A and 3B) and also for a conventional gain element (3C and 3D). Measurements are shown with markers ( $3 \mathrm{~A}$ and $3 \mathrm{C}$ ) and simulation results with solid and dashed lines (3B and 3D). The external cavity geometry is schematically shown in the inset.

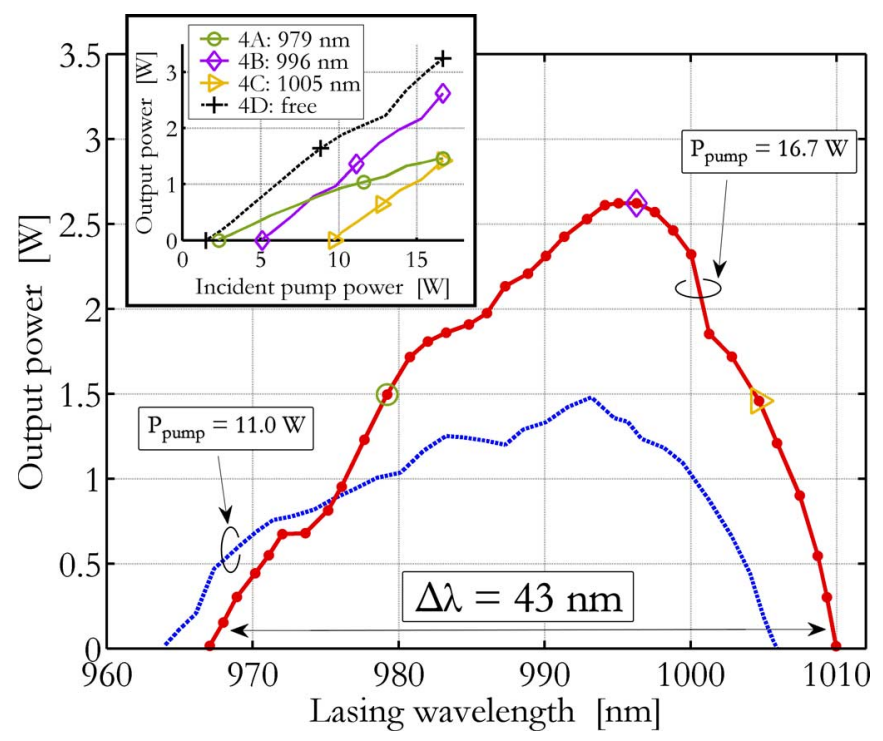

Fig. 4. Measured power spectra of the OP-SDL with broadband gain element at two (high) values for the pump power. The tuning range for $16.7-\mathrm{W}$ incident pump power was $43 \mathrm{~nm}$ and for $11.0 \mathrm{~W}$ it was $42 \mathrm{~nm}$. The inset shows the power characteristics of the OP-SDL at three wavelengths $(4 \mathrm{~A}: \lambda=979 \mathrm{~nm}$; 4B: $\lambda=996 \mathrm{~nm}$; and 4C: $\lambda=1005 \mathrm{~nm}$; also marked in the spectrum) as well as for the free-running OP-SDL without the BRF (4D).

The threshold pump intensity at different lasing wavelengths was measured (see Fig. 3). Also shown are the results from the simulation, which used a measured value for the loss in the BRF ( $\sim 0.1 \%$ per single pass) and the nominal $1 \%$ loss of the outcoupling mirror to calculate the required gain element reflectance, and consequently the threshold pump intensity, using the same model as in the design. Also shown are the corresponding results for a conventionally designed gain element with resonant periodic gain (RPG) with the same nominal center wavelength. Considering the uncertainties in material parameters and errors in epitaxial growth, the agreement between experiment and simulation is good. These results strongly suggest, first, that our un- derlying model is largely correct, and that no uncontrolled factor plays a decisive role in the broadband threshold behavior, and, second, that the optimization indeed significantly increases the accessible tuning range as seen in the simulations.

The broadband properties prevail at high-power operation, as is evident from the output power versus lasing wavelength curves in Fig. 4. In these experiments, the maximum range of continuous tuning was $43 \mathrm{~nm}$, corresponding to a rotation of the BRF by $\sim 8^{\circ}$, and the maximum output power was $2.6 \mathrm{~W}$ for an incident pump power of $16.7 \mathrm{~W}$. During these measurements, the cavity was optimized for high output power and wide tuning range rather than for optimal beam quality, resulting in an output beam of rather poor quality, but this is of little importance for the demonstration of the broadband properties of the gain element.

\section{CONClusion}

High reflectance at a constant pump power in a specified, wide, spectral region was obtained from a parametric optimization of the layer structure of the gain element in an OP-SDL. Direct experimental agreement with simulations demonstrates the effectiveness of the design strategies. The broadband properties prevail under high-power operation-a tuning range of $43 \mathrm{~nm}$ (4.3\% relative) at a maximum output power of $2.6 \mathrm{~W}$ was achieved.

\section{REFERENCES}

[1] J. Sandusky and S. Brueck, "A CW external-cavity surface-emitting laser," IEEE Photon. Technol. Lett., vol. 8, no. 3, pp. 313-315, Mar. 1996.

[2] M. Kuznetsov, F. Hakimi, R. Sprague, and A. Mooradian, "High-power ( $>0.5-\mathrm{W} \mathrm{CW}$ ) diode-pumped vertical-external-cavity surface-emitting semiconductor lasers with circular TEM 00 beams," IEEE Photon. Technol. Lett., vol. 9, no. 8, pp. 1063-1065, Aug. 1997.

[3] B. Rudin et al., "Highly efficient optically pumped vertical-emitting semiconductor laser with more than $20 \mathrm{~W}$ average output power in a fundamental transverse mode," Opt. Lett., vol. 33, no. 22, pp. 2719-2721, Nov. 2008.

[4] J. Chilla, Q. Shu, H. Zhou, E. Weiss, M. Reed, and L. Spinelli, "Recent advances in optically pumped semiconductor lasers," in Proc. SPIE, 2007, vol. 6451, p. 645109.

[5] F. Demaria, S. Lorch, S. Menzel, M. C. Riedl, F. Rinaldi, R. Rosch, and P. Unger, "Design of highly efficient high-power optically pumped semiconductor disk lasers," IEEE J. Sel. Topics. Quantum Electron., vol. 15, no. 3, pp. 973-977, May/Jun. 2009.

[6] L. Fan et al., "Extended tunability in a two-chip VECSEL," IEEE Photon. Technol. Lett., vol. 19, no. 8, pp. 544-546, Apr. 15, 2007.

[7] J. Paajaste, S. Suomalainen, R. Koskinen, A. Härkönen, M. Guina, and M. Pessa, "High-power and broadly tunable GaSb-based optically pumped VECSELs emitting near $2 \mu \mathrm{m}, " J$. Cryst. Growth, vol. 311, no. 7, pp. 1917-1919, Mar. 2009.

[8] A. A. Ukhanov, A. Stintz, P. G. Eliseev, and K. J. Malloy, "Comparison of the carrier induced refractive index, gain, and linewidth enhancement factor in quantum dot and quantum well lasers," Appl. Phys. Lett., vol. 84, no. 7, pp. 1058-1060, Feb. 2004.

[9] A. Garnache, A. A. Kachanov, F. Stoeckel, and R. Houdré, "Diode-pumped broadband vertical-external-cavity surface-emitting semiconductor laser applied to high-sensitivity intracavity absorption spectroscopy," J. Opt. Soc. Amer. B, vol. 17, no. 9, pp. 1589-1598, 2000.

[10] J. Geske, K. Gan, Y. Okuno, J. Piprek, and J. Bowers, "Vertical-cavity surface-emitting laser active regions for enhanced performance with optical pumping," IEEE J. Quantum Electron., vol. 40, no. 9, pp. 1155-1162, Sep. 2004.

[11] R. Häring, R. Paschotta, A. Aschwanden, E. Gini, F. Morier-Genoud, and U. Keller, "High-power passively mode-locked semiconductor lasers," IEEE J. Quantum Electron., vol. 38, no. 9, pp. 1268-1275, Sep. 2002. 